\section{REVISTA BRASILEIRA DE QUALIDADE DE VIDA}

\title{
A Síndrome de Burnout e os níveis de atividade física em policiais militares ambientais de Alagoas, Brasil
}

\section{Burnout syndrome and levels of physical activity in environmental Alagoas military police, Brazil}

\author{
Diogo Ferreira da Rocha \\ Universidade Federal de Alagoas - UFAL - Maceió - Alagoas - Brasil \\ diogosim@hotmail.com \\ Jorge Lopes Cavalcante Neto \\ Universidade do Estado da Bahia - UNEB - Jacobina - Bahia - Brasil \\ jorgelcneto@hotmail.com
}

\section{RESUMO}

OBJETIVO: Verificar a relação entre a Síndrome de Burnout e os níveis de atividade física de policiais militares ambientais do Estado de Alagoas.

MÉTODOS: Trata-se de um estudo descritivo, realizado no Batalhão de Polícia Militar Ambiental de Alagoas, com 30 policiais de ambos os sexos. Aplicou-se um questionário sociodemográfico, o Questionário Internacional de Atividade Física (IPAQ) e o Inventário de Maslach para avaliação de Burnout (MBI). Utilizou-se a estatística descritiva, Odds Ratio (OR) bruto e ajustado com intervalo de confiança (IC) de $95 \%$.

RESULTADOS: Houve um predomínio de indivíduos do sexo masculino, uma frequência superior de militares com carga horária semanal acima de 40 horas semanais, além de que a maioria $(66,7 \%)$ afirmou que a profissão de policial interfere em suas vidas pessoais. Na análise sobre a Síndrome de Burnout, entre as três fases (exaustão emocional, despersonalização e a satisfação com o trabalho), houve um maior escore para exaustão emocional ( $\mathrm{M}=21,20 \pm 6,44 \mathrm{DP})$, que indica altos índices de fadiga. Apesar de exercerem mais de uma atividade laboral, os dados do IPAQ revelou que $60 \%$ dos militares foram enquadrados como ativos, o que pode ter uma relação com a não detecção da Síndrome de Burnout. Contudo, não foram encontradas associações significativas das variáveis de estudo com a utilização do OR.

CONCLUSÕES: Não foram evidenciados possíveis casos da Síndrome de Burnout entre os sujeitos investigados. A maioria dos policiais foi classificada como fisicamente ativos, enquanto que os mesmos evidenciaram que há uma necessidade urgente de melhorias nas condições de trabalho oferecidas no serviço militar, o que implicaria diretamente na melhoria da saúde e qualidade de vida desses militares.

PALAVRAS-CHAVE: Burnout. Atividade física. Policiais Ambientais. 


\section{ABSTRACT}

OBJECTIVE: Verify the relationship between Burnout Syndrome and physical activity levels of environmental military police of the State of Alagoas.

METHODS: This is a descriptive study, conducted in the Environmental military police battalion of Alagoas, with 30 officers of both sexes. Applied a socio-demographic questionnaire, the IPAQ to detect levels of physical activity and the Maslach Burnout inventory (MBI). Descriptive statistics was used, crude Odds Ratio (OR) and adjusted with the confidence interval (CI) of 95\%.

RESULTS: There was a predominance of males, a higher frequency of military with weekly workload over 40 hours per week, plus most (66.7\%) stated that the profession of policeman interferes in their personal lives. On analysis of the Burnout Syndrome among the three phases (emotional exhaustion, depersonalization and satisfaction with work), there was a greater emotional exhaustion to score $(\mathrm{M}=21.20 \pm 6,44 \mathrm{DP})$ that indicates high levels of fatigue. Although exercising more than one labor activity, the IPAQ data revealed that $60 \%$ of the military were framed as assets, which may have a relationship with the non-detection of Burnout Syndrome. No significant associations were found of the variables of study with the use of the OR.

CONCLUSIONS: Possible cases of the Syndrome of Burnout between the subject ones were not evidenced researched. The majority of the police was classified as physically active, whereas the same had evidenced that it has a urgent necessity of improvements in the conditions of work offered military service to it, what would directly imply in the improvement of its health and quality of life of these military.

KEYWORDS: Burnout. Physical activity. Environmental Police.

\section{Introdução}

A qualificação profissional tornou-se uma exigência constante em instituições públicas e privadas. Tal exigência associa-se diretamente com o trabalho, aumentando a pressão por maior produtividade, especialização, principalmente para as profissões que se relacionam diretamente com as pessoas, como a de policiais militares (OLIVEIRA; BARDAGI, 2009). Nessa perspectiva, os efeitos despendidos pelo trabalhador para garantir o emprego, ser valorizado e reconhecido, produz, muitas vezes, efeitos indesejados na saúde dos trabalhadores. Entre os efeitos indesejados algumas patologias, como o estresse e a Síndrome de Burnout (CARLOTTO; CÂMARA, 2008).

$\mathrm{O}$ estresse e a Síndrome de Burnout geram sintomas como a sensação de desgaste físico, cansaço, tensão muscular, problemas com a memória, insônia, baixa autoestima e irritabilidade (OLIVEIRA; BARDAGI, 2009). Por particularidades relacionadas ao trabalho, como risco iminente de morte, carga horária elevada e baixos salários, existem profissões que apresentam maior tendência a essas patologias, com destaque para a dos policiais (GONÇALO et al., 2010), dos médicos (FELICIANO; KOVACS; SARINHO, 2011) e dos professores (ARAÚJO; SAURA, 2009).

A profissão de policial militar é de fundamental importância para a sociedade, possuindo caráter preventivo-repressivo contra a desordem pública, crimes contra a vida, crimes ambientais entre outros. No entanto, devido ao crescimento da violência, principalmente nas grandes cidades, houve um aumento da carga horária de trabalho dos policiais militares que, em situação de risco e submetidos às implicações do regulamento militar, tem sua atuação profissional em condições físicas e psicológicas inadequadas (OLIVEIRA; SANTOS, 2010; SILVA; VIEIRA, 2008),

Além disso, os policiais militares enfrentam os riscos que a profissão oferece àqueles que estão no combate direto (policiais de serviço externo) e indireto (os administrativos), os baixos salários, a visão muitas vezes inadequada que a sociedade é detentora, a falta de investimentos dos governantes e a necessidade de realizar trabalhos extras nos horários de folga para complementar a renda, fazendo com que essa instituição, junto com seus funcionários, não consiga realizar as tarefas 
com eficácia plena (OLIVEIRA; SANTOS, 2010; SPODE; MERLO, 2006). Esses fatores tornam os policiais militares suscetíveis à Síndrome de Burnout, também conhecida como Síndrome do Esgotamento Profissional, e ao estresse (OLIVEIRA; SANTOS, 2010; CARLOTTO; CÂMARA, 2008).

A síndrome apresenta as seguintes sintomatologias: fadiga constante, distúrbio do sono, dores musculares, cefaleias, imunidade diminuída, disfunção sexual, negligência, irritabilidade, agressividade, ideia suicida, desmotivação, ansiedade, ironia, cinismo, depressão e paranoia (BENEVIDES-PEREIRA, 2005).

No contexto da rotina do profissional militar é possível observar baixos níveis de atividade física (MONTEIRO et al., 1998). Os níveis inferiores de atividade física estão relacionados à falta de tempo e à rotina diária dos policiais militares que, normalmente, além do serviço inerente à atividade de militar, fazem trabalhos extras nas horas de folga, deixando de executar atividades físicas na frequência e intensidade recomendadas. Muito se fala sobre os efeitos negativos que a falta de exercício físico pode promover, tais como cardiopatias, diabetes, osteoporose (MOSER; MELO; SANTOS, 2004) e estresse (COSTA et al., 2007). Contudo, os níveis observados em muitos sujeitos que executam atividades que demandariam elevados níveis de atividade física, como policiais militares, são considerados inadequados (MOTA; PEREIRA; TEIXEIRA, 2006; CALAMITA; SILVA FILHO; CAPPUTTI, 2010; JESUS; JESUS, 2010; JESUS; PITANGA, 2011).

Existe uma diferença de conceito de atividade física e exercício físico. A atividade física é qualquer movimento corporal que leve o corpo a níveis acima do repouso. O exercício físico são atividades planejadas que têm como objetivo aprimorar mais de um componente da aptidão física, como: condição aeróbia, força e flexibilidade que estão diretamente relacionados à saúde (ARAÚJO; ARAÚJO, 2000).

A literatura aponta diversos benefícios que a atividade física oferece à saúde (PARDINI et al., 2001), tais como melhora da condição aeróbia, reduzindo os riscos coronarianos (GUEDES; GUEDES, 2001), e melhora do estresse ocupacional (TAMAYO, 2001). Tamayo (2001) descreve a relação de desproporcionalidade da atividade física e o estresse ocupacional, afirmando que quanto melhor o condicionamento físico, menor os níveis de estresse. Além disso, há uma maior tolerância ao estresse em praticantes de atividade física. Justifica-se, assim, a prática regular de atividades e exercícios físicos como fator de proteção para o acometimento de sintomatologias específicas do estresse ocupacional, entre os quais a Síndrome de Burnout.

Nesse cenário, o objetivo do presente estudo foi verificar a relação entre a Síndrome de Burnout e os níveis de atividade física de policiais militares ambientais do Estado de Alagoas.

\section{Métodos}

Trata-se de um estudo descritivo, realizado no Batalhão de Polícia Militar Ambiental de Alagoas, localizado na BR 316 - Km 14, Clima Bom II, Maceió-Al, região Nordeste do Brasil. O local é cercado por mata atlântica e possui um campo de futebol para prática de atividade física, além de uma piscina semiolímpica desativada. Os pesquisadores receberam autorização do local para poder mencioná-lo no estudo.

Foram incluídos policiais com no mínimo 5 anos de serviço na polícia militar e que estavam a mais de 2 e menos de 25 anos no Batalhão de Polícia Ambiental. Foram excluídos aqueles que se encontravam de férias ou licença especial no período de aplicação dos questionários.

A população do estudo foi composta de 60 policiais que preencheram os critérios de inclusão. Contudo, devido a não autorização por parte de 10 militares e a não localização de 20 sujeitos durante o período de realização do estudo, a amostra final, constituída por acessibilidade, foi composta por $50 \%$ da população alvo, ou seja, 30 policiais que consentiram participar da pesquisa assinando o Termo de Consentimento Livre e Esclarecido (TCLE).

Foram aplicados três questionários: (i) o sociodemográfico, elaborado para a análise de características relativas ao contexto familiar e laboral dos respondentes; (ii) o Questionário 
Internacional de Atividade Física (IPAQ), para a análise do grau de atividade física dos respondentes, baseados em perguntas que foram validadas em grau de reprodutibilidade e confiabilidade em relação aos testes em aparelhos, permitindo classificar os sujeitos em muito ativos, ativos, irregularmente ativos e sedentários (PARDINI et al., 2001); (iii) o Inventário de Maslach para avaliação de Burnout, que usa escores de 0 a 6 para aferir o grau de proximidade ou afastamento em relação à Síndrome de Burnout, relacionando o grau de satisfação com o trabalho e com os companheiros de profissão. $\mathrm{O}$ instrumento é dividido nas seguintes categorias: exaustão emocional, realização pessoal com o trabalho e despersonalização. Adicionalmente é apresentado o escore total. Quanto mais próximo de 100, maiores as chances de o sujeito apresentar a Síndrome de Burnout (CARLOTTO; CÂMARA, 2008).

A coleta de dados aconteceu de acordo disponibilidade do efetivo nas instalações do Batalhão de Polícia Ambiental.

Utilizou-se a análise estatística descritiva, com frequências relativas e absolutas, médias e desvios padrões, além do cálculo da razão de chances (Odds Ratio) bruto e ajustado com Intervalo de Confiança (IC) de $95 \%$. Para a realização de todos os cálculos estatísticos foi utilizado o programa SPSS versão 20.0 para Windows.

A presente pesquisa seguiu todas as prerrogativas da Declaração de Helsinque e da Resolução 466/12 CNS/MS para procedimentos de pesquisa com seres humanos, tendo sido aprovado o protocolo desta pesquisa pelo Comitê de Ética da Universidade Federal de Alagoas (UFAL), com parecer número 439.380 .

\section{Resultados}

A amostra final do estudo foi de 30 policiais militares do Batalhão Ambiental do Estado de Alagoas. A Tabela 1 descreve as principais informações sociodemográficas e ocupacionais do grupo de policiais participantes do presente estudo. Cabe destacar que a maioria dos sujeitos foi do sexo masculino, trabalhavam mais de 40 horas semanais e frequentemente realizavam horas extras. 
Tabela 1 - Variáveis sociodemográficas e ocupacionais de policiais militares ambientais, Alagoas, 2014 (N=30)

\begin{tabular}{|c|c|c|}
\hline Variável & $\mathbf{N}$ & $\%$ \\
\hline \multicolumn{3}{|l|}{ Sexo } \\
\hline Masculino & 24 & 80,0 \\
\hline Feminino & 06 & 20,0 \\
\hline \multicolumn{3}{|l|}{ Faixa etária } \\
\hline Até 30 anos & 03 & 10,0 \\
\hline De 31 a 40 anos & 11 & 36,7 \\
\hline De 41 a 50 anos & 16 & 53,3 \\
\hline \multicolumn{3}{|l|}{ Estado civil } \\
\hline Solteiro & 08 & 26,7 \\
\hline Casado & 22 & 73,3 \\
\hline \multicolumn{3}{|l|}{ Escolaridade } \\
\hline Ensino médio incompleto & 01 & 03,3 \\
\hline Ensino médio completo & 10 & 33,3 \\
\hline Superior incompleto & 07 & 23,3 \\
\hline Superior completo & 07 & 23,3 \\
\hline Pós-graduação & 05 & 16,8 \\
\hline \multicolumn{3}{|l|}{ Posto ou graduação } \\
\hline Soldado & 10 & 33,3 \\
\hline Cabo & 09 & 30,0 \\
\hline Sargento & 11 & 36,7 \\
\hline \multicolumn{3}{|l|}{ Trabalho extra } \\
\hline Sim & 08 & 26,6 \\
\hline Não & 22 & 73,4 \\
\hline \multicolumn{3}{|l|}{ Remuneração } \\
\hline Até 3 salários mínimos & 05 & 16,7 \\
\hline De 3 a 8 salários mínimos & 25 & 83,3 \\
\hline \multicolumn{3}{|l|}{ Tempo de serviço } \\
\hline Entre 5 e 10 anos & 10 & 33,3 \\
\hline Entre 11 e 15 anos & 02 & 06,7 \\
\hline Entre 16 a 20 anos & 04 & 13,3 \\
\hline Entre 21 e 35 anos & 14 & 46,7 \\
\hline \multicolumn{3}{|c|}{ Horas de serviço por semana } \\
\hline Mais de 40 horas & 26 & 86,6 \\
\hline 40 horas & 02 & 06,7 \\
\hline Entre 30 e 40 horas & 02 & 06,7 \\
\hline \multicolumn{3}{|l|}{ Turnos de trabalho } \\
\hline Dia & 05 & 16,7 \\
\hline Dia/Noite & 25 & 83,3 \\
\hline \multicolumn{3}{|l|}{ Horas extras } \\
\hline Sempre & 06 & 20,0 \\
\hline Frequentemente & 10 & 33,3 \\
\hline Raramente & 08 & 26,7 \\
\hline Nunca & 06 & 20,0 \\
\hline \multicolumn{3}{|l|}{ Afastamento do trabalho } \\
\hline Nenhum & 23 & 76,7 \\
\hline 1 a 2 dias & 05 & 16,7 \\
\hline 3 a 7 dias & 01 & 03,3 \\
\hline 8 dias ou mais & 01 & 03,3 \\
\hline
\end{tabular}

Ao se observar as características gerais de saúde dos policiais, descritas na Tabela 2, chama a atenção o elevado percentual de profissionais que afirmaram que a profissão de militar interfere em sua vida pessoal $(66,7 \%)$. Por outro lado, cabe destacar que os sujeitos apontam a necessidade de iniciativas do próprio Batalhão de Polícia para que práticas previstas em lei possam ser efetivadas, como a criação e manutenção de programas de exercícios físicos e atendimento psicossocial no local de trabalho, o que poderia refletir diretamente na melhoria da saúde e da qualidade de vida destes profissionais. 
Tabela 2 - Características gerais de saúde e qualidade de vida de policiais militares ambientais, Alagoas, 2014 (N=30)

\begin{tabular}{|c|c|c|}
\hline Variável & $\mathbf{N}$ & $\%$ \\
\hline \multicolumn{3}{|l|}{ Uso de tabaco } \\
\hline Sim & 02 & 06,6 \\
\hline Não & 28 & 93,4 \\
\hline \multicolumn{3}{|l|}{ Uso de álcool } \\
\hline Sim & 15 & 50,0 \\
\hline Não & 15 & 50,0 \\
\hline \multicolumn{3}{|l|}{ Consumo de álcool semanal } \\
\hline Não bebo & 15 & 50,0 \\
\hline 1 a 7 doses & 11 & 36,6 \\
\hline 8 a 14 doses & 02 & 06,7 \\
\hline 15 ou mais doses & 02 & 06,7 \\
\hline \multicolumn{3}{|l|}{ Uso de preservativo } \\
\hline Nunca & 07 & 23,3 \\
\hline Eventualmente & 12 & 40,0 \\
\hline Sempre & 11 & 36,7 \\
\hline \multicolumn{3}{|l|}{ A profissão interfere na vida pessoal } \\
\hline Sim & 20 & 66,7 \\
\hline Não & 10 & 33,3 \\
\hline \multicolumn{3}{|l|}{ Percepção de estado de saúde } \\
\hline Excelente & 03 & 10,0 \\
\hline Bom & 21 & 70,0 \\
\hline Regular & 06 & 20,0 \\
\hline \multicolumn{3}{|l|}{ Relacionamento com pessoas } \\
\hline Excelente & 11 & 36,7 \\
\hline Bom & 17 & 56,7 \\
\hline Regular & 02 & 06,6 \\
\hline \multicolumn{3}{|l|}{ Sentimento em relação à vida } \\
\hline Muito bem & 10 & 33,3 \\
\hline Bem & 19 & 63,4 \\
\hline Mal & 01 & 03,3 \\
\hline \multicolumn{3}{|l|}{ Sentiu dores nos trabalhos } \\
\hline Nunca & 11 & 36,7 \\
\hline 1 a 2 dias & 14 & 46,7 \\
\hline 3 a 7 dias & 03 & 10,0 \\
\hline 8 ou mais dias & 02 & 06,6 \\
\hline \multicolumn{3}{|l|}{ Como o BPM ajudaria em sua qualidade de vida } \\
\hline Receber mais informações & 07 & 23,4 \\
\hline Participar de um programa de exercícios físicos & 09 & 30,0 \\
\hline Ter horário flexível para frequentar academia & 04 & 13,3 \\
\hline Receber incentivo da empresa & 06 & 20,0 \\
\hline Participar de cursos e palestras & 01 & 03,3 \\
\hline Usar instalações de clube ou academia & 03 & 10,0 \\
\hline
\end{tabular}

Fonte: Autoria própria (2014).

A Tabela 3 descreve as médias dos escores do Inventário de Maslach para avaliação de Burnout nas três categorias descritas no instrumento. Observou-se que dentre as categorias, a maior média foi encontrada na categoria exaustão emocional $(M=21,20 \pm 6,44)$. Apesar do escore do total geral ter sido superior a 50, não foi possível apontar no presente estudo que os militares entrevistados estejam sofrendo da Síndrome de Burnout.

Tabela 3 - Médias e desvios-padrão das categorias do Inventário de Maslach para avaliação do Burnout e escore total dos policiais militares ambientais de Alagoas, $2014(\mathrm{~N}=30)$

\begin{tabular}{lcc}
\hline $\begin{array}{c}\text { Categorias do Inventário de Maslach para } \\
\text { avaliação de Burnout }\end{array}$ & Médias & Desvio-padrão \\
\hline Exaustão emocional & 21,20 & $\pm 6,44$ \\
Realização pessoal com o trabalho & 19,47 & $\pm 5,05$ \\
Despersonalização & 12,23 & $\pm 1,06$ \\
Total & 52,90 & $\pm 13,91$ \\
\hline
\end{tabular}

Fonte: Autoria própria (2014). 
A Tabela 4 expõe as frequências dos níveis de atividade física dos policiais militares ambientais participantes do estudo. Observou-se que a maioria dos sujeitos foi classificada como Ativo (60\%), segundo as classificações do IPAQ.

Tabela 4 - Frequências relativas e absolutas dos níveis de atividade física, segundo as classificações do IPAQ, em policiais militares ambientais, Alagoas, $2014(\mathrm{~N}=30)$

\begin{tabular}{lcc}
\hline Classificações do IPAQ & $\mathbf{N}$ & $\mathbf{\%}$ \\
\hline Muito Ativo & 07 & 23,3 \\
Ativo & 18 & 60,0 \\
Irregularmente Ativo & 04 & 13,4 \\
Sedentário & 01 & 03,3 \\
\hline \multicolumn{2}{c}{ Fonte: Autoria própria (2014). }
\end{tabular}

Ao se realizar possíveis associações utilizando-se o cálculo da razão de chances (Odds Ratio) das variáveis sociodemográficas, ocupacionais e de saúde com os níveis de atividade física e a Síndrome de Burnout não foram encontradas diferenças significativas entre o grupo de militares ambientais do Estado de Alagoas.

\section{Discussão}

O presente estudo se propôs verificar a relação entre a Síndrome de Burnout e os níveis de atividade física de policiais militares ambientais do Estado de Alagoas, visando reunir informações importantes acerca da saúde e qualidade de vida de sujeitos que lidam com a segurança ambiental do Estado de Alagoas. A partir da aplicação do Inventário Maslach para avaliação de Burnout, observouse um escore total igual a 52,90 $( \pm 13,98 \mathrm{DP})$. Com esses resultados não foi possível afirmar que foram encontrados casos de Burnout entre os militares. Já entre as categorias descritas no Inventário para avaliação do Burnout, o maior escore encontrado foi no quesito exaustão emocional $(\mathrm{X}=21,20 \pm 6,44 \mathrm{DP})$. Aspecto esse que, no caso de militares, se caracteriza pela fadiga intensa (OLIVEIRA, 2011). Oliveira (2011) aponta alguns fatores para o acometimento da fadiga intensa entre os militares, como uma carga horária de trabalho elevada, baixos salários, com uma rotina de atividade física no trabalho abaixo do recomendado, pressão da mídia e imposições previstas em regulamento da polícia militar, que podem levar a problemas de saúde mental.

Os militares possuem fatores laborais propícios a desenvolver a Síndrome de Burnout por estarem envolvidos diretamente com problemas pessoais dos cidadãos, normalmente pessoas desconhecidas, que ao interagir com características de personalidade de cada profissional poderá colaborar para que ocorra ou não o Burnout, pois, algumas pessoas possuem um nível de resiliência maior que outras, apresentando assim maior suscetibilidade ao desenvolvimento da patologia (BENEVIDES-PEREIRA, 2005).

Ainda sobre a atividade policial e a Síndrome de Burnout, Oliveira (2011) não encontrou em seu estudo uma relação com o trabalho e o desenvolvimento da patologia, mas sim com um alto nível de estresse. Para Oliveira e Bardagi (2009), a exposição prolongada a fatores estressantes no ambiente de trabalho pode levar ao Burnout. Diante desse contexto, alternativas válidas que possam suscitar potenciais fatores protetores ao estresse relativo ao trabalho estão sendo cada vez mais discutidos na literatura (JODAS; HADDAD, 2009; TIRONI et al., 2009). Um dos principais fatores é a prática regular de atividade física. Assim, manter-se ativo fisicamente é um fator de proteção para acometimento de problemas de estresse no trabalho, como a Síndrome de Burnout.

Segundo a classificação IPAQ, os indivíduos, em sua maioria (60\%), encontraram-se Ativos, demonstrando que apesar das longas jornadas de trabalho, muitas vezes em mais de um lugar, os policiais conseguem manter-se fisicamente ativos. Esse nível da atividade física pode estar relacionado com o não aparecimento de sintomas severos da Síndrome de Burnout ou até a minimização dos efeitos da mesma (SOUSA; PINHEIRO, 2013). Bardagi e Oliveira (2009) colocam que um programa de exercícios físicos regulares, alimentação adequada e descanso necessário servem para minimizar o efeito dos fatores causadores de estresse e Burnout. 
Ainda sobre a necessidade de atividade física, Calheiros, Cavalcante Neto e Calheiros (2013), em estudo realizado com policiais militares alagoanos, apontam a necessidade de um programa de condicionamento físico na instituição policial e de uma boa aptidão física para os militares exercerem a profissão, já que esses profissionais desenvolvem serviços de grande dispêndio energético, como embarcar e desembarcar de viatura, carregar equipamentos de proteção como coletes balísticos, armamento, além do uniforme. Além disso, o papel da atividade física bem planejada e orientada vai além do desenvolvimento de boa aptidão física, ela pode ser responsável pela melhoria dos níveis de estresse, beneficiando a saúde e qualidade de vida desses profissionais.

Dentre as variáveis ocupacionais analisadas, chamou atenção à elevada parcela dos indivíduos $(66,7 \%)$ que afirmaram que o seu trabalho de policial militar interfere na vida pessoal. Para Calheiros, Cavalcante Neto e Calheiros (2013), a influência da profissão policial militar na vida pessoal normalmente leva os policiais a terem problemas de saúde e de relacionamento, produzidos com a insegurança que existe dentro da profissão e o risco iminente de morte presente no labor.

Costa et al. (2007), em estudo com policiais militares do Rio Grande do Norte, destacaram que a profissão policial é uma das mais estressantes, pois, os mesmos envolvem-se constantemente em situações de alto risco e tensão. Como forma de tentar minimizar essa tensão ocupacional, os militares investigados faziam uso de bebidas alcoólicas, o que apresentava relação com um estilo de vida não saudável e uma maior predisposição aos possíveis efeitos do Burnout. Consoante aos resultados obtidos neste estudo observou-se que o uso de álcool entre os militares ambientais de Alagoas teve um valor expressivo. Contudo, o principal apontamento do estudo se referiu a elevada frequência de policiais considerados fisicamente ativos, o que possivelmente aponta uma tendência desse ser um fator de proteção para os baixos escores encontrados das fases do Burnout entre esses militares.

Monteiro et al. (1998), em estudo sobre atividade física e fatores socioeconômicos e ocupacionais em policiais militares de São Paulo, apontam a importância da atividade física para um bom desempenho da profissão policial militar. Os autores relacionam a ausência dessas atividades com a falta de uma política interna e de tempo para que os militares desenvolvam as atividades nas horas de folga e no trabalho.

\section{Conclusão}

Não foram evidenciados possíveis casos da Síndrome de Burnout entre os militares ambientais investigados. Dentre as categorias do Inventário Maslach para avaliação de Burnout, a exaustão emocional teve destaque dentre as demais categorias, indicando potenciais fatores estressantes no ambiente laboral dos sujeitos investigados.

Apesar das longas jornadas de trabalho, das horas extras e das duplas ou triplas jornadas laborais, evidenciou-se que os militares ambientais foram em, sua maioria, considerados ativos, segundo as classificações do IPAQ.

Os resultados do presente estudo foram divulgados no local de coleta de dados, com sigilo da identidade dos sujeitos investigados, em forma de seminário, contribuindo para que a prática regular de atividade física possa ser cada vez mais difundida entre esses profissionais dentro e fora do ambiente de trabalho.

\section{Referências}

ARAÚJO, A. M. N. F. L. de; SAURA, C. J. I. Relação do exercício físico com o stress, com os factores de stress e com o Burnout em docentes portugueses. Efdeportes - Revista Digital, Buenos Aires, ano 14, n. 131, abr. 2009. Disponível em <http://www.efdeportes.com/efd131/relacao-doexercicio-fisico-com-o-stress-em-docentes.htm>. Acesso em: 22 out. 2013. 
ARAÚJO, D. S. M. S. de; ARAÚJO, C. G. S de. Aptidão física, saúde e qualidade de vida relacionada à saúde em adultos. Revista Brasileira de Medicina do Esporte, Rio de Janeiro, v. 6, n. 5, p. 194203, set./out. 2000. Crossef

BENEVIDES-PEREIRA, A. M. T. A Síndrome de Burnout. Saúde Mental no Trabalho, Goiânia, V. 1, p. 36-51, 2005.

CALAMITA, Z.; SILVA FILHO, C. R.; CAPPUTTI, P. F. Fatores de risco para doenças cardiovasculares no policial militar. Revista Brasileira de Medicina do Trabalho, São Paulo, v. 8, n. $1, \quad$ p. $39-45, \quad 2010 . \quad$ Disponível em: <http://www.anamt.org.br/site/upload_arquivos/revista_brasileira_de_medicina_do_trabalho__volume_8_n\%C2\%BA_1_20122013132158533424.pdf>. Acesso em: 27 fev. 2014.

CALHEIROS, D. dos S.; CAVALCANTE NETO, J. L.; CALHEIROS, D. dos S. A qualidade de vida e os níveis de atividade física de policiais militares de Alagoas, Brasil. Revista Brasileira de Qualidade de Vida, Ponta Grossa, v. 5, n. 1, p. 59-71, jul./set. 2013. Crossef

CARLOTTO, M. S.; CÂMARA, S. G. Análise da produção científica sobre a Síndrome de Burnout no Brasil. Psico, v. 32, n. 2, p. 152-158, abr./jun. 2008. Disponível em <http://revistaseletronicas.pucrs.br/ojs/index.php/revistapsico/article/view/1461>. Acesso em: 22 maio 2013.

COSTA, M.; ACCIOLY JÚNIOR, H.; OLIVEIRA, J.; MAIA, E. Estresse: diagnóstico dos policiais militares em uma cidade brasileira. Revista Panamericana de Salud Publica, Washington, v. 21, n. 4, p. 217-222, abr. 2007. Crossef

FELICIANO, K. V. de O.; KOVACS, M. H.; SARINHO, S. W. Burnout entre médicos da Saúde da Família: os desafios da transformação do trabalho. Ciência \& Saúde Coletiva, v. 16, n. 8, p. 33733382, 2011. Disponível em: <http://www.scielo.br/pdf/csc/v16n8/a04v16n8.pdf>. Acesso em: 15 jan. 2014.

GONÇALO, H.: RUI GOMES, A.; BARBOSA, F.; AFONSO, J. Stresse ocupacional em forças de segurança: um estudo comparativo. Análise Psicológica, v. 1, n. 28, p. 165-178, 2010. Disponível em $\langle$ http://www.scielo.oces.mctes.pt/pdf/aps/v28n1/v28n1a12.pdf $>$. Acesso em: 30 jan. 2014.

GUEDES, D. P, GUEDES, J. E. R. P. Atividade física, aptidão cardiorrespiratória, composição da dieta e fatores de risco predisponentes às doenças cardiovasculares. Arquivos Brasileiros de Cardiologia, v. 77, n. 3, p. 243-250, 2001. Disponível em: <http://publicacoes.cardiol.br/abc/2001/7703/7703005.pdf>. Acesso em: 22 jan. 2014.

JESUS, C. C. B.; PITANGA, C. P. S. Nível de atividades físicas dos Policiais Militares da 53 ${ }^{\mathrm{a}}$ CIPM em Mata de São João - Bahia. Revista do Curso de Educação Física - UNIJORGE, v. 01, n. 01, p. 25-36, jul./dez. 2011. Disponível em: <http://revistas.unijorge.edu.br/corpomovimentosaude/pdf/artigo2011_2_artigo25_36.pdf>. Acesso em: 24 fev. 2014.

JESUS, G. M.; JESUS, E. F. A de. Predisposição para desenvolver resistência insulínica em policiais militares. Pensar a Prática, v. 13, n. 02, p. 1-15, 2010. Disponível em: <http://www.revistas.ufg.br/index.php/fef/article/viewArticle/9644>. Acesso em: 23 fev. 2014. 
JODAS, D. A.; HADDAD, M. C. L. Síndrome de Burnout em trabalhadores de enfermagem de um pronto socorro de hospital universitário. Acta Paulista de Enfermagem, São Paulo, v. 22, n. 2, p. 192-197, mar./abr. 2009. Crossef

MONTEIRO, H. L.; GONÇALVES, A.; PADOVANI, C. R.; FERMINO NETO, J. L. Fatores sócioeconômicos e ocupacionais e a prática de atividade física regular: estudo a partir de policiais militares em Bauru, São Paulo. Motriz, v. 4, n. 2, p. 91-97, dez. 1998. Disponível em <http://www.rc.unesp.br/ib/efisica/motriz/04n2/4n2_ART3.pdf>. Acesso em: 30 jan. 2014.

MOSER, D. C.; MELO, S. I. L.; SANTOS, S. G. dos. Influência da atividade física sobre a massa óssea de mulheres. Revista Brasileira de Cineantropometria \& Desempenho Humano, v. 6, n. 1, p. 46-53, 2004. 2 Disponível em <https://periodicos.ufsc.br/index.php/rbcdh/article/view/3840/16825>. Acesso em: 02 fev. 2014.

MOTA, C. B.; PEREIRA, E. F. P.; TEIXEIRA, C. S. Práticas esportivas e o serviço militar. Revista Mineira de Educação Física, Viçosa, v. 14, n. 1, p. 7-18, 2006. Disponível em: <http://www.revistamineiradeefi.ufv.br/artigos/arquivos/446921e7e4956d9e23b2abeafdc70ef6.pdf > . Acesso em: 27 fev. 2014.

OLIVEIRA, D. R. Atividade policial e sua relação com a Síndrome de Burnout. Revista Eficaz Revista Científica Online, Maringá, v. 1, n. 1, p. 1- 16, jan. 2011.

OLIVEIRA, K. L. de; SANTOS, L. M. dos. Percepção da saúde mental em policiais militares da força tática e de rua. Sociologias, Porto Alegre, v. 12, n. 25, p. 224-250, set./dez. 2010. rossef

OLIVEIRA, P. L. M. de; BARDAGI, M. P. Estresse e comprometimento com a carreira em policiais militares. Boletim de Psicologia, São Paulo, v. 59, n. 131, dez. 2009. Disponível em: $<$ http://pepsic.bvsalud.org/scielo.php?pid=S0006-59432009000200003\&script=sci_arttext $>$. Acesso em: 02 fev. 2014.

PARDINI, R.; MATSUDO, S.; ARAÚJO, T.; MATSUDO, V.; ANDRADE, E.; BRAGGION, G.; ANDRADE, D., OLIVEIRA, L.; FIGUEIRA JR., A.; RASO, V. Validação do questionário internacional de nível de atividade física (IPAQ- versão 6): estudo piloto em adultos jovens brasileiros. Revista Brasileira de Ciência e Movimento, Brasília, v. 9, n. 3, p. 45-51, jul. 2001. Disponível em <http://portalrevistas.ucb.br/index.php/RBCM/article/viewArticle/393 >. Acesso em: 01 fev. 2014.

REINHOLD, H. H. O sentido da vida: prevenção de stress e Burnout do professor. 2004. 189f, Tese (Doutorado em Psicologia) - Programa de Pós-Graduação em Psicologia do Centro de Ciências da Vida da Pontifícia Universidade Católica de Campinas (PUC), Campinas.

SILVA, M. B. da; VIEIRA, S. B. O processo de trabalho do militar estadual e a saúde mental. Saúde e Sociedade, São Paulo, v. 17, n. 4, p. 161-170, out./dez. 2008. Crossef

SOUSA, A. R.; PINHEIRO, A. de O. Correlação entre a síndrome de Burnout e nível de atividade física em professores da rede pública de Uruará/PA. In: ENCONTRO DE PÓS-GRADUAÇÃO E INICIAÇÃO CIENTÍFICA - UNIVERSIDADE CAMILO CASTELO BRANCO. 2013, São José dos Campos. Anais... São José dos Campos: Unicastelo, 2013. Disponível em: 〈http://unicastelo.br/epginic/arquivos/anais/epg/ciencias_da_saude/235\%20-\%20EPG307.pdf>.

Acesso em: 21 fev. 2014. 
SPODE, C. B.; MERLO, A. R. C. Trabalho policial e saúde mental: uma pesquisa junto aos Capitães da Polícia Militar, Psicologia: Reflexão e Crítica, Porto Alegre, v. 19, n. 3, p. 362-370, 2006. crossef

TAMAYO, A. Prioridades axiológicas, atividade física e estresse ocupacional. Revista de Administração Contemporânea, v. 5, n. 3, p. 127-147, set./dez. 2001. rrossef

TIRONI, M. O. S.; NASCIMENTO SOBRINHO, C. L.; BARROS, D. S.; REIS. E. J. F. B.; MARQUES FILHO, E. S.; ALMEIDA, A.; BITENCOURT, A.; FEITOSA, A. I. R.; NEVES, F. S.; MOTA, I. C. C.; FRANÇA, J.; BORGES, L. G.; LORDÃO, M. B. J.; TRINDADE, M. V.; TELES, M. S.; ALMEIDA, M. B. T.; SOUZA, Y. M. Trabalho e síndrome da estafa profissional (Síndrome de Burnout) em médicos intensivistas de Salvador. Revista da Associação Médica Brasileira, São Paulo, v. 55, n. 6, p. 656-662, 2009. Crossef 